\title{
Mire Vegetation Database of Latvia
}

\author{
Liene Aunina
}

\begin{abstract}
The TURBOVEG database Mire Vegetation Database of Latvia (GIVD ID EU-LV-002) comprises 1927 relevés described in mires of Latvia by the author since 1995 and is annually updated. The plot size is $1-4 \mathrm{~m}^{2}$. Percentage cover of Vascular plants and bryophytes were evaluated for each sample plot. It also includes 156 relevés on vegetation changes in pine forests after selective cutting and heath mowing in Erica tetralix habitats. This is a private database. The data are mainly used for preparation of mire vegetation classification system of Latvia. So far the database covers two classes Phragmito-Magnocaricetea and ScheuchzerioCaricetea nigrae. When Vegetation Database of Latvia at Laboratory of Geobotany of Institute of Biology will be created it will be part of national vegetation database.
\end{abstract}

Keywords: Eastern Europe; fen; tall-sedge vegetation.

GIVD Database ID: EU-LV-002

Last update: $2012-05-05$

\section{Mire Vegetation Database of Latvia}

Scope: The main scope of the database was mire vegetation classification in Latvia. So far it covers two classes Phragmito-Magnocaricetea and Scheuchzerio-Caricetea nigrae.

$\begin{array}{ll}\text { Status: emerging Period: 1995-2010 } & \end{array}$

Database manager(s): Liene Aunina (Isalmina@latnet.lv)

Owner: Liene Aunina (private)

Web address: [NA]

Availability: according to a specific agreement

Database format(s): TURBOVEG

Publication: [NA]

Plot type(s): normal plots; time series

Non-overlapping plots: 2,083

Total plot observations: 2,083

Online upload: no Online search: no
Export format(s): TURBOVEG, Excel, CSV file, plain text file

Export format(s): TURBOVEG, Excel, CSV file, plain text file

Plot-size range: $1-4 \mathrm{~m}^{2}$

\begin{tabular}{ll} 
Estimate of existing plots: 2,083 & Completeness: $100 \%$ \\
\hline Number of sources: $[\mathrm{NA}]$ & Valid taxa: 330
\end{tabular}

Countries: LV: $100.0 \%$

Forest: $7 \%$ - Non-forest: aquatic: $0 \%$; semi-aquatic: $93 \%$; arctic-alpine: $0 \%$; natural: $0 \%$; semi-natural: $0 \%$; anthropogenic: $0 \%$

Guilds: all vascular plants: $100 \%$; bryophytes (terricolous or aquatic): $100 \%$

Environmental data: soil depth: $3 \%$

Performance measure(s): cover: $100 \%$

Geographic localisation: GPS coordinates (precision $25 \mathrm{~m}$ or less): $100 \%$

Sampling periods: $1990-1999: 75.0 \%$; 2000-2009: $23.0 \% ; 2010-2019: 2.0 \%$

Information as of 2012-07-12; further details and future updates available from http://www.givd.info/ID/EU-LV-002

Liene Aunina (lsalmina@ latnet.lv)

Laboratory of Geobotany, Institute of Biology, 3 Miera Street, LV-2169 Salaspils, LATVIA 